\title{
Quantification of hand muscle volume and composition in patients with rheumatoid arthritis, psoriatic arthritis and psoriasis
}

Andreas Friedberger ${ }^{1 *}$, Camille Figueiredo ${ }^{2}$, Alexandra Grimm', Isabelle d'Oliveira ${ }^{2}$, Tobias Bäuerle ${ }^{3}$, Jürgen Rech², Arnd Kleyer ${ }^{2}$, David Simon², Michael Uder ${ }^{3}$, Georg Schett $^{2}$ and Klaus Engelke ${ }^{2}$

\begin{abstract}
Background: Psoriasis (PsO), psoriatic arthritis (PsA) and rheumatoid arthritis (RA) are inflammatory diseases. PsA and RA are characterized by bone and muscle loss. In RA, bone loss has been extensively characterized, but muscle loss has, to the best of our knowledge, not been quantified to date.

Methods: A random forest based segmentation method was used to analyze hand muscle volume in T1 weighted MRI images of 330 patients suffering from Pso, PsA or RA. In addition, fat volume was quantified using MRI Dixon sequences in a small subset $(n=32)$.

Results: Males had a higher relative muscle volume than females (14\% for Pso, $11 \%$ for PsA, n.s. for RA). Between 40 and 80 years male Pso patients lost 13\%, male PsA patients 16\%, male RA patients 23\% and female PsA patients $30 \%$ of their relative muscle volume. After adjustment for age, relative muscle volume in males RA patients was $16 \%$ and in female RA patients 9\% lower than in Pso patients. In male RA patients relative muscle volume was 13\% lower in than in male PsA patients. There was no difference in females. A significant negative correlation $\left(R^{2}=0.18\right)$ between relative intramuscular fat content relative hand muscle volume was observed.

Conclusion: These preliminary data showed that relative hand muscle volume significantly decreased with age in male and female patients with Pso, PsA and RA patients. Independent of age, relative hand muscle volume was significantly smaller in patients with RA compared to the patients with Pso and the difference was twice as large in males compared to females. Also in male but not in female RA patients relative hand muscle volume was significantly smaller than in PsA patients.
\end{abstract}

Keywords: Psoriasis, Psoriatic arthritis, Rheumatoid arthritis, Magnetic resonance imaging, Random forest based segmentation, Hand muscle, Fat

\footnotetext{
* Correspondence: andreas.friedberger@imp.uni-erlangen.de

'Institute of Medical Physics, University of Erlangen-Nuremberg, Henkestraße 91, 91052 Erlangen, Germany

Full list of author information is available at the end of the article
}

(c) The Author(s). 2020 Open Access This article is licensed under a Creative Commons Attribution 4.0 International License, which permits use, sharing, adaptation, distribution and reproduction in any medium or format, as long as you give appropriate credit to the original author(s) and the source, provide a link to the Creative Commons licence, and indicate if changes were made. The images or other third party material in this article are included in the article's Creative Commons licence, unless indicated otherwise in a credit line to the material. If material is not included in the article's Creative Commons licence and your intended use is not permitted by statutory regulation or exceeds the permitted use, you will need to obtain permission directly from the copyright holder. To view a copy of this licence, visit http://creativecommons.org/licenses/by/4.0/. The Creative Commons Public Domain Dedication waiver (http://creativecommons.org/publicdomain/zero/1.0/) applies to the data made available in this article, unless otherwise stated in a credit line to the data. 


\section{Background}

Rheumatoid arthritis (RA) is a chronic inflammatory disease, which is associated with bone and cartilage loss [1]. About two thirds of RA patients also suffer from rheumatoid cachexia (RC) [1], an accelerated involuntary loss of fat-free skeletal muscle mass, which is larger than the decrease related to 'normal' aging (sarcopenia). The term RC has already been coined in 1992 [2], but etiology and pathophysiology of RC are still not well understood. RC is underdiagnosed and undertreated [3-5].

Structural bone damage of RA has been assessed using radiographs, magnetic resonance imaging (MRI) and more recently high resolution peripheral quantitative computed tomography (HR-pQCT). In contrast, techniques to quantify muscle properties are still under development. For the diagnosis of $\mathrm{RC}$, advanced muscle and fat imaging is required. $85 \%$ of RA subjects have normal BMI [6] because the muscle loss is often compensated by fatty infiltration, resulting in normal body weight. Thus, the sole use of BMI for the diagnosis of $\mathrm{RC}$ is misleading.

It has been suggested $[7,8]$ that the increased fatty infiltration may be one factor to explain the discrepancy of age related decreases between muscle mass and volume in healthy subjects [9]. In addition, adipose tissue is a source of pro-inflammatory cytokines, which triggers inflammatory responses $[10,11]$, an important observation with potentially high clinical relevance not only in RA but also in psoriatic arthritis (PsA) and psoriasis (Pso). Similar to RA, PsA is also characterized by inflammation of the synovial tissue, which ultimately results in bone, cartilage and muscle damage. However, the production and secretion of pro-inflammatory cytokines is lower than in RA $[12,13]$, which may explain why PsA is usually less destructive. Pso mainly affects the skin, but subclinical joint inflammation has also been reported $[14,15]$. Nevertheless, to the best of our knowledge, no major impact of Pso on muscle has been reported so far.

This study specifically addresses the assessment of hand muscle volume and composition in patients with rheumatoid, psoriatic arthritis and psoriasis using standard clinical and Dixon MRI sequences. The primary study aim was to compare hand muscle volume among the three diseases, independent of age and BMI. Additionally, preliminary results of a hand fat volume assessment were included. For the purpose of this study, psoriasis patients were used as controls.

\section{Methods}

\section{Patient details}

In this study we used existing MRI scans of the dominant hand of 330 ambulatory care patients diagnosed with RA, PsA or Pso, from the Department of Medicine 3 of the University of Erlangen-Nuremberg. Apart from the disease there were no additional inclusion or exclusion criteria. BMI data was available from 206 patients. This subset will be denoted as $\mathrm{S}_{\mathrm{BMI}}$.

\section{MR imaging}

Routine MRI Scans were performed with a 1.5-Tesla MRI system (MAGNETOM Aera, Siemens Healthcare $\mathrm{GmbH}$, Erlangen, Germany). Subjects were positioned in prone position with head first. For signal reception, a hand/wrist radiofrequency 16-channel coil was used. The standard clinical MR protocol included several sequences from which a fat suppressed $\mathrm{T}_{1 \mathrm{w}}$ TSE was selected for analysis. More recently, a $\mathrm{T}_{2 \mathrm{w}}$ Dixon TSE sequence has been added to the clinical protocol. The specifications of the two sequences used for this study are summarized in Table 1.

\section{Image processing}

The image processing consisted of pre-processing of the $\mathrm{T} 1$ scans, segmentation using the $\mathrm{T} 1$ scans and transformation of the segmentation results to the Dixon fat fraction scans.

The T1 weighted MR scans were pre-processed with the N4ITK algorithm [16, 17] to remove artificial intensity modulations caused by inhomogeneous magnetic fields (Fig. 1). The segmentation of the hand and of hand muscles was performed in these homogenized images. This step required knowledge of the hand cross sectional area (CSA), which was obtained as a series of 2D contours determined for each slice by thresholding and other basic image processing steps.

The core of the muscle segmentation was a random forest classifier. Random forest is a well-known ensemble learning method from machine learning [18], which is widely used for image segmentation [19]. The random forest classifies each voxel into muscle or background based on image features in the voxel neighborhood. The combination of all voxels classified as muscle constitutes the muscle volume of interest (VOI). The classifier has to be trained beforehand using manually segmented hand scans. The final segmentation step was a Gaussian fit of the histogram of the muscle VOI. Voxels outside the range of the mean \pm 2 standard deviations were removed. This removed erroneously included hypointense ligaments and tendons or hyperintense vessels. The results were manually edited by the operator, if necessary. The top panel in Fig. 2 shows a typical segmentation result.

For the quantification of muscle fat content, a 2 ptDixon technique was used [20]. This sequence produces a water $I_{\text {water }}$ and a fat image $I_{\text {fat }}$, from which a quantitative fat fraction image $I_{\mathrm{ff}}$ can be calculated: 
Table 1 Specifications of the T1 and the Dixon sequences as used in this study. TSE stands for turbo spin echo, TR for the repetition time, TE for the echo time and TD for the time difference between spin and gradient echo formation

\begin{tabular}{|c|c|c|c|c|c|c|c|}
\hline \multirow[t]{2}{*}{ Sequence } & \multicolumn{2}{|c|}{ In-plane matrix } & \multicolumn{2}{|l|}{ Slices } & \multirow{2}{*}{$\begin{array}{l}\text { TR } \\
\text { (ms) }\end{array}$} & \multirow{2}{*}{$\begin{array}{l}\text { TE } \\
\text { (ms) }\end{array}$} & \multirow{2}{*}{$\begin{array}{l}\text { TD } \\
\text { (ms) }\end{array}$} \\
\hline & Voxel count & Resolution $\left(\mathrm{mm}^{2}\right)$ & Count & Thickness(mm) & & & \\
\hline $\mathrm{T}_{1 w}$ TSE & $320 \times 320$ & $0.5 \times 0.5$ & 64 & 3.0 & 760 & 13 & 0 \\
\hline $\mathrm{T}_{2 w}$ Dixon TSE & $320 \times 320$ & $0.5 \times 0.5$ & 30 & 3.0 & 3040 & 78 & 0 \\
\hline
\end{tabular}

$$
I_{f f}=\frac{I_{\text {fat }}}{I_{\text {water }}+I_{\text {fat }}} * 1000
$$

$\mathrm{I}_{\mathrm{ff}}$ assigns a percentage of fat (Fig. 2 bottom) to each voxel. In $\mathrm{I}_{\mathrm{ff}}$, grey values range from 0 (meaning the voxel consists of $0 \%$ fat) to 1000 (meaning the voxel consists of $100 \%$ fat), i.e. 1 grey value equals $0.1 \%$ fat. In order to restrict the fat analysis to muscle, the muscle VOI determined in the T1 dataset was transferred to $\mathrm{I}_{\mathrm{ff}}$. For this purpose, the hand surface was registered from the T1 dataset to $\mathrm{I}_{\mathrm{ff}}$ via rigid multimodal image registration. The resulting transformation was then applied to the muscle VOI (Fig. 2 bottom).

\section{Output parameters}

Direct output parameters calculated from the T1 images were hand $\left(\mathrm{V}_{\mathrm{H}}\right)$ and muscle volume $\left(\mathrm{V}_{\mathrm{M}}\right)$, derived between user defined proximal and distal ends of the metacarpal bone III (MCP; Fig. 3). These were the accumulated volumes of all voxels participating in the hand resp. muscle segmentation. From these two parameters the relative muscle volume $\left(V_{M}{ }^{\text {rel }}=V_{M} / V_{H}\right)$ was calculated. Output parameters from $\mathrm{I}_{\mathrm{ff}}$ were absolute fat volume $\left(V_{F}\right)$ and relative fat content $\left(V_{f}^{\text {rel }}=V_{F} / V_{M}\right)$ within the muscle VOI. $V_{F}$ was the accumulated volume of all voxels participating in the hand muscle segmentation times their individual fat fraction values $\mathrm{I}_{\mathrm{ff}}$.

The segmentation reliability was obtained as reanalysis precision errors. Three operators analyzed 14 randomly chosen data sets once (inter-operator) and one operator analyzed these 14 data sets three times (intra-operator). Reanalysis precision errors were calculated as root mean square average of standard deviation and coefficient of variation of individual data sets [21].

\section{Statistics}

In order to evaluate the dependence of hand and relative muscle volume on age and BMI, a linear regression with

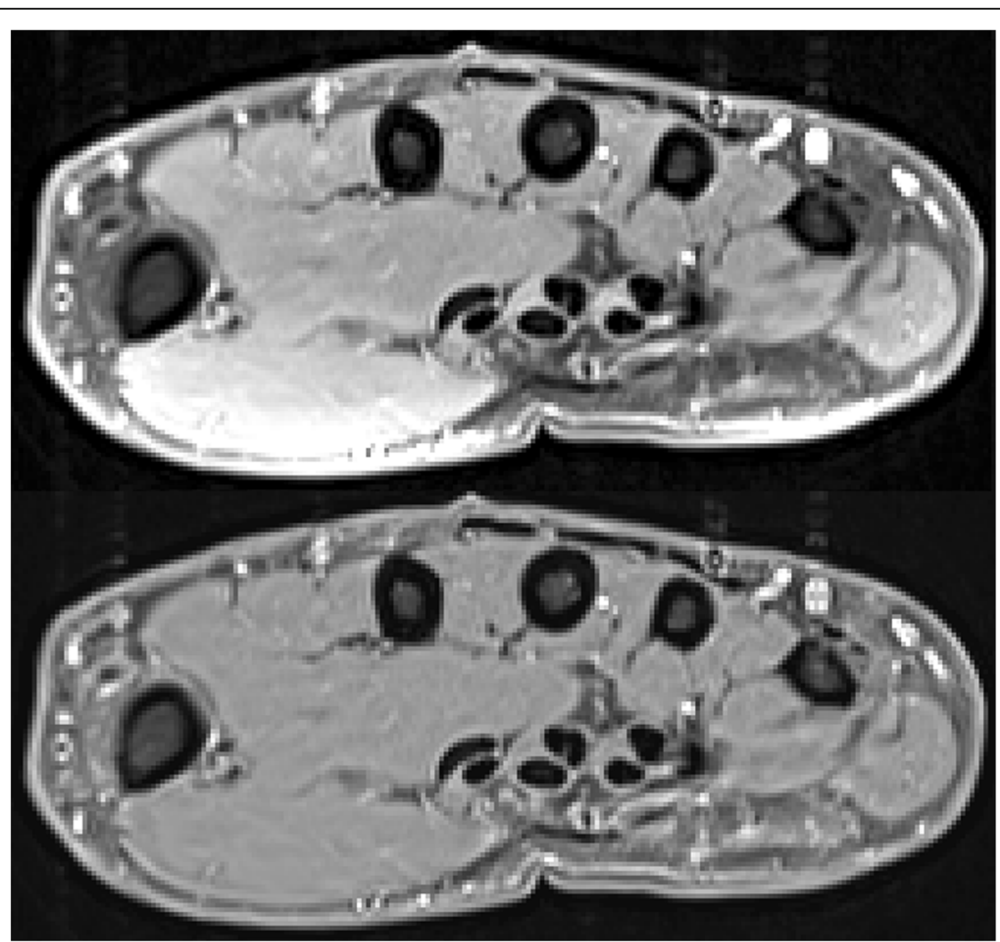

Fig. 1 Top: Axial slice of a T1 weighted fat suppressed MRI hand scan in the metacarpal region. Notice the inhomogeneous intensity distribution of the grey values caused by a bias field, especially in the thenar region (lower left). Bottom: Same slice but with removed bias field by nonuniform intensity normalization (N4ITK) 


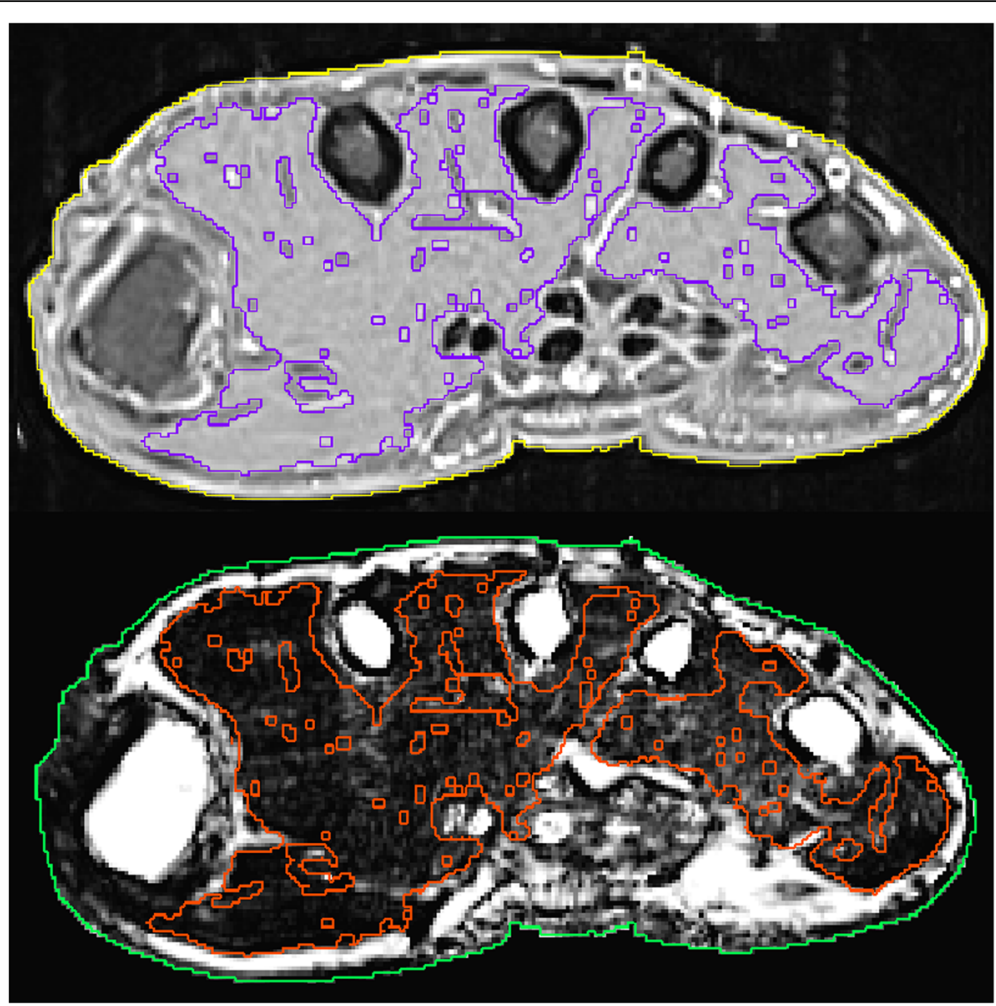

Fig. 2 Top: Axial slice with segmented cross sectional area (yellow) and muscle (purple). Bottom: The green CSA and the red muscle segmentation were imported from the processed $\mathrm{T} 1$ image via multimodal image registration

age and BMI as independent variables was used. For the assessment of differences of $\mathrm{V}_{\mathrm{H}}$ and $\mathrm{V}_{\mathrm{M}}{ }^{\text {rel }}$ among the three groups corresponding to patients with RA, PsA or Pso, the factor disease was added as independent categorical variable to the particular linear model. Group differences, denoted as $\Delta$, were the average difference over the specified age intervals. Since some studies showed an association between hand grip strength and age $^{3}$ [22], age was substituted by age $\mathrm{e}^{3}$ in a separate linear regression model, under the assumption that hand muscle volume is correlated to hand grip strength.

For a more age-sensitive group difference assessment, the relative muscle volume was partitioned into 10 year intervals from the 5th to the 8th decade. For each decade, group differences were tested using 1-way Anova and post-hoc Tuskey HSD. Further, linear regressions with independent variables age, BMI and hand and relative muscle volume were performed for $\mathrm{V}_{\mathrm{F}}$ and $\mathrm{V}_{\mathrm{F}}{ }^{\text {rel }}$. Since the number of patients, for which fat data were available, was small $(n=32)$, the fat analysis was not stratified for sex or disease.

$p$ values below 0.05 were considered significant. All statistics were performed with the statistics software $\mathrm{R}$ (version 3.2.5, [23]).

\section{Results}

Patient age is summarized in Table 2. Dixon imaging based fat fraction measurements were available from 32 patients, since just recently added to the clinical protocol. This subset will be denoted as $\mathrm{S}_{\text {dixon }}$. Inter- and intra-reanalysis precision was excellent (Table 3 ).

\section{Assessment of hand volume}

Males had a higher hand volume (37\% for Pso, $34 \%$ for PsA, 48\% for RA) than females. The dependence of $V_{H}$ on age is displayed in Fig. 4. In males, $\mathrm{V}_{\mathrm{H}}$ was correlated positively with age in the Pso $(p=0.02)$ and negatively in the RA group $(p=0.05)$. In females, a positive correlation between $\mathrm{V}_{\mathrm{H}}$ and age was found for Pso $(p=0.01)$ and RA $(p<0.001)$ patients. For PsA patients there was no significant correlation between $\mathrm{V}_{\mathrm{H}}$ and age, neither for men nor for women. Between 20 and 80 years, hand volume of males decreased by $17 \%$ in RA patients, increased by $9 \%$ in Pso patients and did not change in PsA patients. In females hand volume increased by $24 \%$ in RA and Pso patients and did not change in PsA patients. After adjustment for age alone or for age and BMI, most group differences in $V_{H}$ were not significant. These results are listed in Table 4 , with $\Delta$ as the $V_{H}$ difference between the two groups, on average in age between 20 


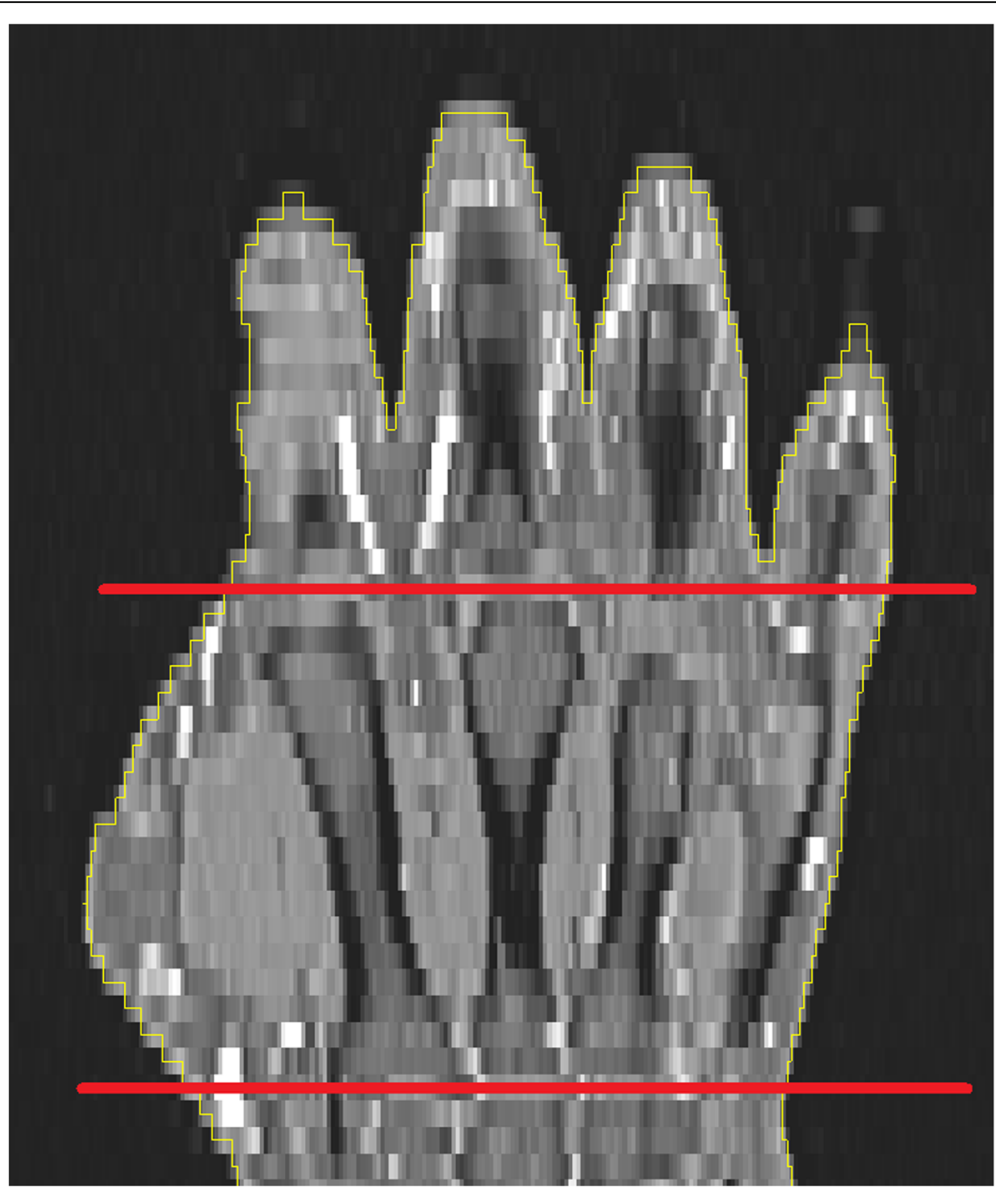

Fig. 3 Coronal slice of a hand MR with segmented CSA (yellow). MCP II to MCP IV are clearly visible. Proximal and distal boundaries of MCP III are marked in red, the analysis is limited to the region in between

and 80. Adjustment for age ${ }^{3}$ instead of age did not significantly change the results.

\section{Assessment of relative muscle volume}

Males had a higher relative muscle volume than females ( $14 \%$ for Pso, $11 \%$ for PsA, n.s. for RA). The dependence of $\mathrm{V}_{\mathrm{M}}^{\text {rel }}$ on age is shown in Fig. 5. The models using age $^{3}$ instead of age better described the age-dependence of $\mathrm{V}_{\mathrm{M}}$ rel. When combining data of all three groups, in males the model using age ${ }^{3}$ explained $24 \%$ of the variance of $\mathrm{V}_{\mathrm{M}}{ }^{\text {rel }}$, compared to $19 \%$ for the model linear in age. In females the model using age ${ }^{3}$ explained $11 \%$ and the linear one $9 \%$ of the variance of $\mathrm{V}_{\mathrm{M}}{ }^{\text {rel }}$. However,

Table 2 Age, sex and diagnosis distribution of the cohort

\begin{tabular}{|c|c|c|c|c|c|c|}
\hline & \multicolumn{2}{|c|}{ Pso $(n=101)$} & \multicolumn{2}{|c|}{$\mathrm{PsA}(n=137)$} & \multicolumn{2}{|c|}{ RA $(n=92)$} \\
\hline & male & female & male & female & male & female \\
\hline$n$ & 63 & 38 & 61 & 76 & 41 & 51 \\
\hline Age (y) & $48 \pm 12$ & $54 \pm 15$ & $56 \pm 14$ & $56 \pm 11$ & $62 \pm 12$ & $60 \pm 14$ \\
\hline
\end{tabular}

differences between independent variables age or age $\mathrm{e}^{3}$ were not observed when comparing the age dependence of $\mathrm{V}_{\mathrm{M}}{ }^{\text {rel }}$ among groups. Therefore group differences in $\mathrm{V}_{\mathrm{M}}{ }^{\text {rel }}$ will only be reported using models linear in age.

Between 20 and 80 years, negative correlations of relative hand muscle volume with age were observed for male PsA $(p=0.01)$ and RA patients $(p<0.001)$ and for female PsA patients $(\mathrm{p}<0.001)$. Correlations for the other groups were not significant. Male PsA patients lost $16 \%$, male RA patients $27 \%$ and female PsA patients $30 \%$ of their relative muscle volume. When restricting the analysis to the range between 40 and 80 years, a negative correlation of relative hand volume with age was also observed for male Pso patients $(\mathrm{p}=0.01)$. Between 40 and 80 years male Pso patients lost $13 \%$, male PsA patients $16 \%$, male RA patients $23 \%$ and female PsA patients $30 \%$ of their relative muscle volume.

Group differences after adjustment for age alone or for age and BMI are shown in Table 5. The $\Delta$ stands for the $\mathrm{V}_{\mathrm{M}}{ }^{\text {rel }}$ difference between the two groups, on average in the denoted age intervals. Between 20 and 80 years, 
Table 3 Reanalysis precision of hand and muscle volume (measured in the T1 image) and of muscle fat content and ratio (measured in the Dixon images) Precision are shown as root mean square average of the standard deviation displayed in the unit of the variable and of the coefficient of variation displayed in \%

\begin{tabular}{|c|c|c|c|c|c|c|c|c|}
\hline \multirow[b]{2}{*}{ Interoperator } & \multicolumn{2}{|c|}{ Hand volume } & \multicolumn{2}{|c|}{ Muscle volume } & \multicolumn{2}{|l|}{ Fat content } & \multicolumn{2}{|c|}{ Fat fraction } \\
\hline & $5.0 \mathrm{~mm}^{3}$ & $0.19 \%$ & $1.5 \mathrm{~mm}^{3}$ & $0.24 \%$ & $364.4 \mathrm{~mm}^{3}$ & $0.60 \%$ & $0.09 \%$ & $0.07 \%$ \\
\hline Intraoperator & $3.4 \mathrm{~mm}^{3}$ & $0.13 \%$ & $0.35 \mathrm{~mm}^{3}$ & $0.05 \%$ & $41.6 \mathrm{~mm}^{3}$ & $0.10 \%$ & $0.006 \%$ & $0.04 \%$ \\
\hline
\end{tabular}

relative muscle volume in males RA patients was $16 \%$ lower and in females $9 \%$ lower than in Pso patients. In males but not in females there was also a significant difference between RA and PsA patients, relative muscle volume was $13 \%$ lower in RA patients.

BMI alone did not show significant correlations with $\mathrm{V}_{\mathrm{M}}{ }^{\text {rel }}$ in either sex. However, after additional adjustment for BMI, in females the difference $\mathrm{V}_{\mathrm{M}}{ }^{\text {rel }}$ between RA and Pso patients was no longer significant, while in males the difference between PsA and Pso patients became significant $(p=0.05)$.

Results were similar in the age group 40-80 years but in males there was no significant difference, between PsA and Pso patients even after adjustment for BMI, while the difference became significant in females.

Results of $\mathrm{V}_{\mathrm{M}}^{\text {rel }}$ by age decade are shown in Fig. 6 for males and Fig. 7 for females. For males, there was a consistent pattern of numerically lower relative hand muscle volume in RA patients for all decades. Significant differences of $\mathrm{V}_{\mathrm{M}}{ }^{\text {rel }}$ among groups in males were observed for the 5th decade (RA-Pso, $p=$ 0.04 , mean difference $=-0.046$ ), 6th decade (RA-Pso, $\mathrm{p}=0.04$, mean difference $=-0.039$ ) and 7th decade (RA-PsA, $\mathrm{p}=0.04$, mean difference $=-0.03$ ) but not for the 8th decade. In females, group differences were not significant for any decade.

\section{Assessment of muscle fat}

Absolute intramuscular fat volume $\left(\mathrm{V}_{\mathrm{F}}\right)$ was positively correlated with hand volume $\mathrm{V}_{\mathrm{H}}\left(\mathrm{R}^{2}=0.74, p<0.0001\right)$. Age, disease and BMI did not significantly impact the correlation.

Relative intramuscular fat content $\left(\mathrm{V}_{\mathrm{F}}^{\text {rel }}\right)$ showed no significant correlation with hand volume but a significant negative correlation with $\mathrm{V}_{\mathrm{M}}{ }^{\text {rel }}\left(\mathrm{R}^{2}=0.18, p=0.004\right)$ (Fig. 8).

\section{Discussion}

In this study we compared hand muscle volume in patients with Pso, PsA and RA. For the purpose of this study subjects with Pso were regarded as control group as Pso patients show only little to no inflammation in the hand joints.

We applied a combination of T1-weighted and 2 ptDixon imaging sequences and a random forest based segmentation method to analyze muscle volume. Hand muscle fat infiltration could only be obtained in a small subset so far. The excellent precision of MR 2 pt-Dixon for fat quantification had been demonstrated earlier [24]. In the same study a high correlation of fat content between 2 pt-Dixon measurements of phantoms and MR spectroscopy, the standard method for fat quantification was reported.
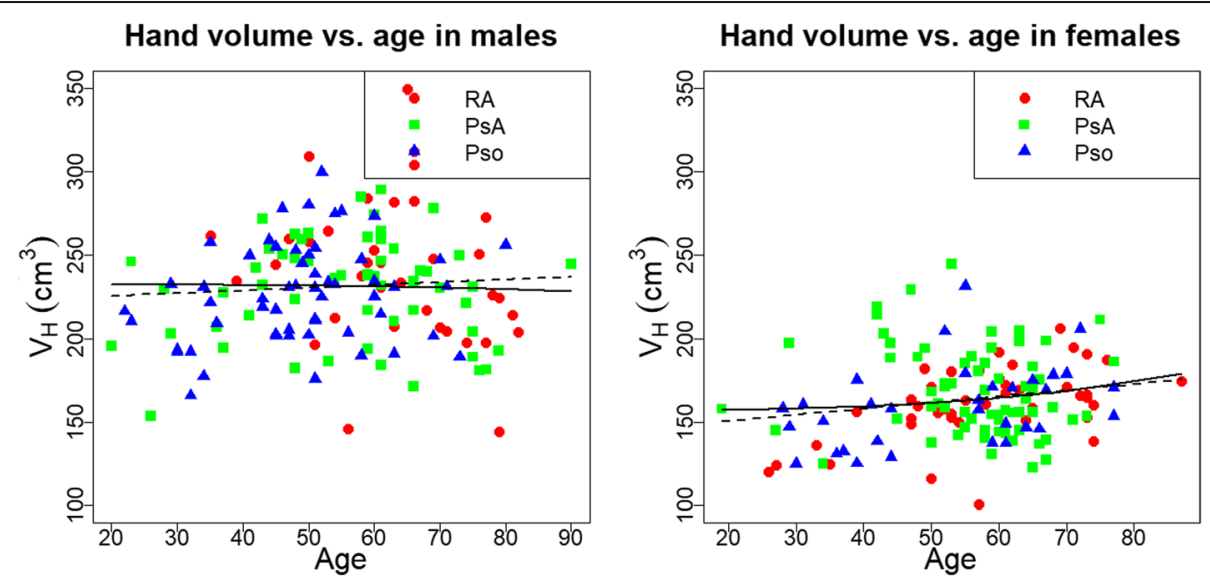

Fig. 4 Age dependency of hand volume for males (left) and females (right). Additionally displayed are the regression line of the model linear (solid black line) and cubic in age (dashed black line) for the union of the three groups 
Table 4 Differences in hand volume between RA, Pso and PsA patients after adjustment for age (left column) and age and BMI (right column) as independent covariates

\begin{tabular}{lll}
\hline$V_{H} /$ males & age & $\begin{array}{l}\text { age \& BMl } \\
\left(\mathrm{S}_{\text {BMI }}\right)\end{array}$ \\
\hline RA - Pso & $\begin{array}{l}p=0.08 \\
\Delta=+14\end{array}$ & n.s. \\
PSA - PsO & n.s. & n.s. \\
RA - PSA & n.s. & $p=0.003 \Delta=+25$ \\
$V_{H} /$ females & & \\
RA - PsO & n.s. & n.s. \\
PSA - PsO & n.s. & $p=0.05 \Delta=-0.13$ \\
RA - PSA & $p=0.07 \Delta=-8.5$ & n.s. \\
\hline
\end{tabular}

$\Delta$ denotes the $V_{H}$ difference (measured in $\mathrm{cm}^{3}$ ) between two groups, averaged over age. Data after age and BMI adjustment were only obtained in subgroup $S_{B M I}$

The central findings of this study showed that the age related decrease of relative hand muscle volume in males was larger than in females (Table 5 and Figs. 6 and 7) and that in males but not in females relative muscle volume was lower in RA than in PsA or Pso patients, independent of age. Results did not significantly change when substituting age with age ${ }^{3}$.

Not surprisingly, males had a higher hand volume than females. Gender differences of relative muscle volume were smaller but with the exception of subjects with RA, males also had higher relative muscle volume than females. In the control (Pso) group, age related decreases were larger for relative muscle than for hand volume, which can be explained by increasing muscle fat infiltration with age. Our very preliminary and still limited results, which showed an inverse relation between relative fat and relative muscle volume support this hypothesis.

A detailed investigation of age related changes per disease group and age decade showed that in the male control group decreases in relative muscle volume started in the sixth decade, indicating progressive sarcopenia. There were no significant difference between the control group and PsA patients for either decade. In males with RA, $\mathrm{V}_{\mathrm{M}}{ }^{\text {rel }}$ was lower than for the two other groups for all age decades and the decline of $\mathrm{V}_{\mathrm{M}}{ }^{\text {rel }}$ was larger, in particular in the 8th decade. This decrease in $V_{M}{ }^{\text {rel }}$ is further amplified by the age related decrease in hand volume showing the strong association between muscle deterioration and RA in male subjects. Differences in relative muscle volume among Pso, PsA and RA female patients were numerically smaller and not significant for most age decades.

The results shown in Figs. 6 and 7 were confirmed after age adjustment in the pooled age groups (Table 5). In males and females, differences in $\mathrm{V}_{\mathrm{M}}{ }^{\text {rel }}$ were significant between RA and Pso patients but age adjusted differences were about twice as high in males. It is speculative but perhaps this larger difference in $V_{M}{ }^{\text {rel }}$ between male RA patients and controls could explain why in males but not in females the difference between RA and PsA groups was also significant. In other words, the main difference in $\mathrm{V}_{\mathrm{M}}{ }^{\text {rel }}$ between males and females is observed in the RA group. Whether this can be explained by the degree of inflammation, by differential therapeutic interventions, differences in the efficacy of an intervention or the way the hands are used in daily life is an interesting questions that warrants further investigation. Unfortunately the power of our data was insufficient to further explore the differential contribution
Rel. muscle volume vs. age in males

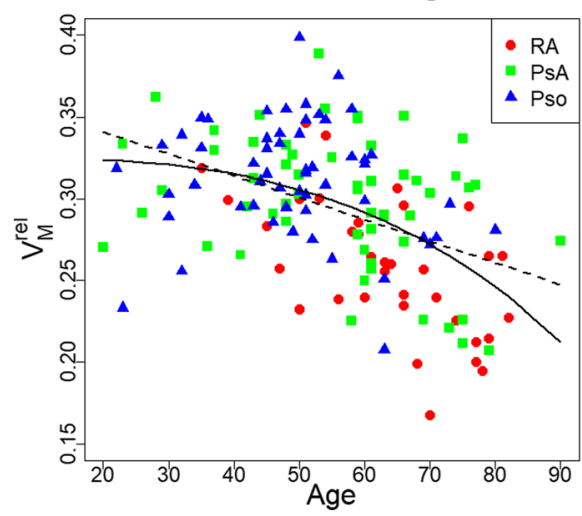

Rel. muscle volume vs. age in females

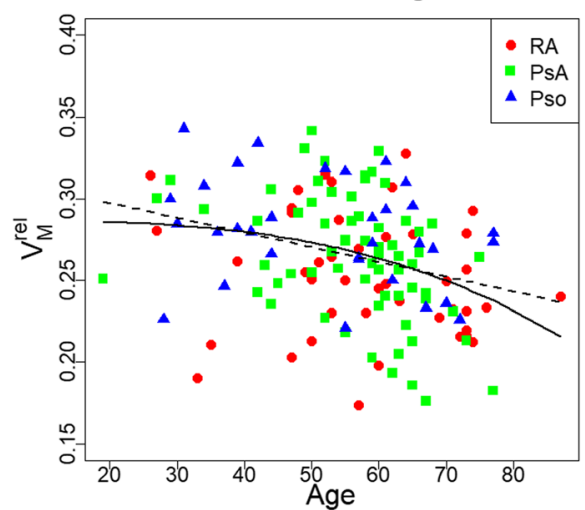

Fig. 5 Age dependency of relative muscle volume for males (left) and females (right). Additionally displayed are the regression line of the model linear (solid black line) and cubic in age (dashed black line) for the union of the three groups 
Table 5 Differences in relative muscle volume between RA, Pso and PsA patients after adjustment for age and age and BMI as independent covariates

\begin{tabular}{|c|c|c|c|c|}
\hline \multirow[b]{2}{*}{$V_{M}{ }^{\text {rel } / \text { males }}$} & \multicolumn{2}{|l|}{ Age $20-80$ years } & \multicolumn{2}{|l|}{ Age $40-80$ years } \\
\hline & age & $\begin{array}{l}\text { age \& BMI } \\
\left(\mathrm{S}_{\text {BMI }}\right)\end{array}$ & age & $\begin{array}{l}\text { age \& BMI } \\
\left(S_{B M I}\right)\end{array}$ \\
\hline RA - Pso & $p<0.0001 \Delta=-0.040$ & $p<0.001 \Delta=-0.045$ & $p<0.0001 \Delta=-0.041$ & $p<0.0001 \Delta=-0.039$ \\
\hline PsA - PsO & n.s. & $p=0.05 \Delta=-0.021$ & n.s. & n.s. \\
\hline RA - PsA & $p<0.0001 \Delta=-0.033$ & $p=0.02 \Delta=-0.023$ & $p<0.0001 \Delta=-0.045$ & $p=0.02 \Delta=-0.024$ \\
\hline \multicolumn{5}{|l|}{$V_{M}{ }^{\text {rel }} /$ females } \\
\hline RA - Pso & $p=0.01 \Delta=-0.023$ & $p=0.09 \Delta=+0.020$ & $p=0.03 \Delta=-0.022$ & $p=0.03 \Delta=-0.020$ \\
\hline PsA - PsO & n.s. & n.s. & n.s. & $p=0.05 \Delta=-0.019$ \\
\hline RA - PsA & n.s. & n.s. & n.s. & n.s. \\
\hline
\end{tabular}

$\Delta$ denotes the $V_{M}{ }^{\text {rel }}$ difference between two groups, on average in the denoted age interval. $V_{M}{ }^{\text {rel }}$ ranges between 0 and 1 , thus $\Delta^{*} 100$ stands for the percentage point difference of relative muscle volume. Data after age and BMI adjustment were only obtained in subgroup $\mathrm{S}_{\mathrm{BMI}}$. The calculations are repeated for the age group 40-80 years (two most-right columns)

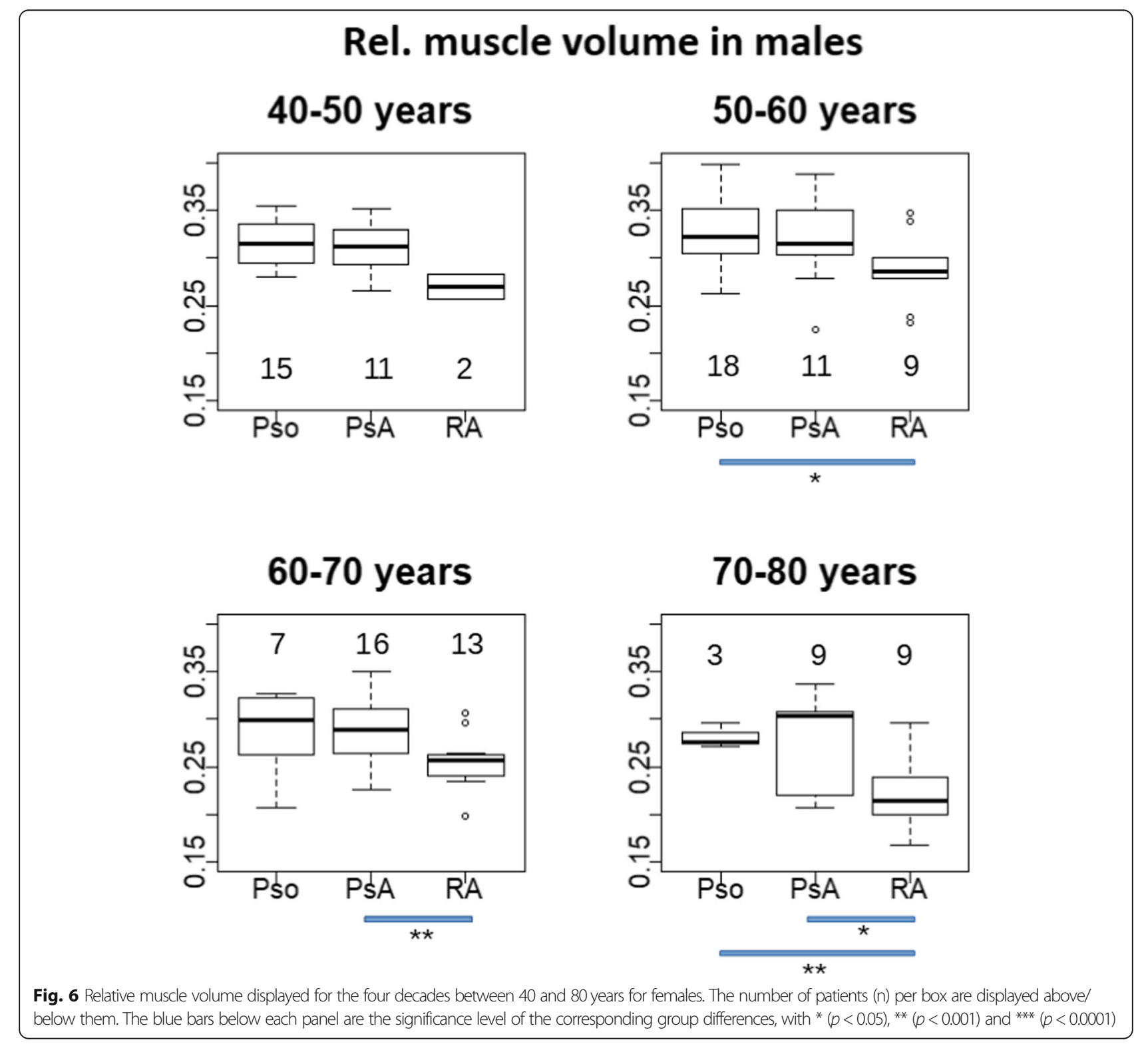




\section{Rel. muscle volume in females}

40-50 years

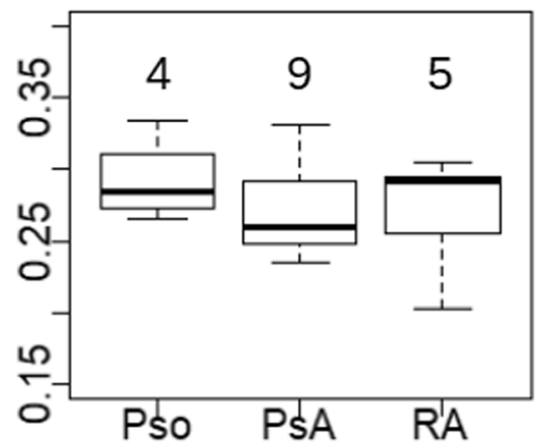

60-70 years

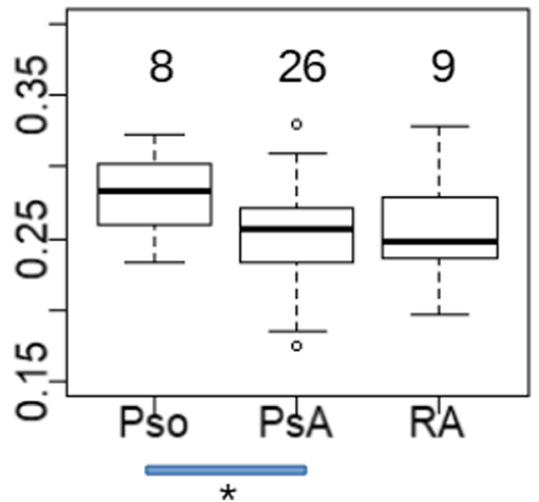

$50-60$ years

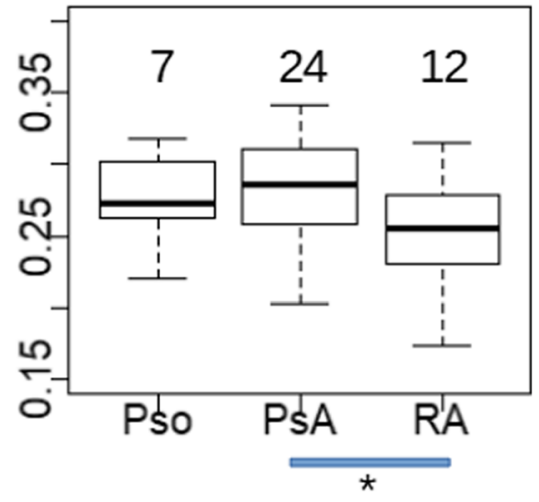

$70-80$ years

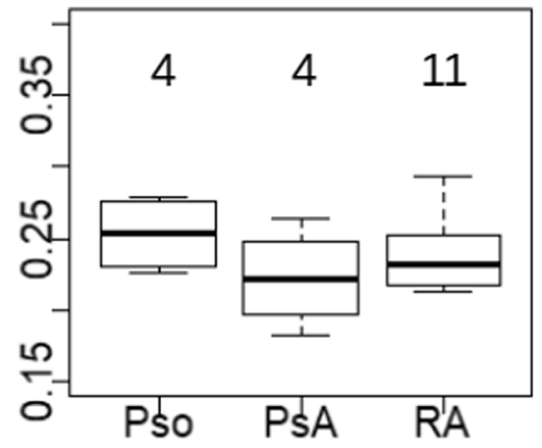

Fig. 7 Relative muscle volume displayed for the four decades between 40 and 80 years for males. For the description of the numbers and blue bars see Fig. 6

of intramuscular adipose tissue in male and female RA patients.

As already indicated in the background section, most RA patients have a normal BMI. This fact was indirectly confirmed in this study. No or only very small, mostly non-significant effects of BMI were observed on $\mathrm{V}_{\mathrm{H}}$, $\mathrm{V}_{\mathrm{M}}{ }^{\text {rel }}$ and $\mathrm{V}_{\mathrm{F}}{ }^{\text {rel }}$. Probably the hand, which was the focus of this study is too small in order to show a significant impact on BMI, which is a whole body parameter. However, BMI also did not reflect changes in fat content caused by fatty infiltration in paraspinal [25], or gluteus muscles [26].

This study was a pilot study to demonstrate that MRI can be used to assess hand and hand muscle volume and intramuscular adipose tissue and to demonstrate differences between patients with Pso, PsA and RA. There are a number of limitations that should be addressed in future investigations:

First, the use of Pso patients as controls could be criticized as recently subclinical inflammation has been reported in Pso patients [15]. Thus a control group of healthy subjects should also be investigated. Second, functional measurements such as grip strength, data on pharmaceutical interventions and other diagnostic scores should be added as covariates to the analysis. Third, fat measurements should be generated in a larger number of subjects. Fourth, this was a cross-sectional study, correlations with age need cautious interpretations. Longitudinal data still have to be collected. 


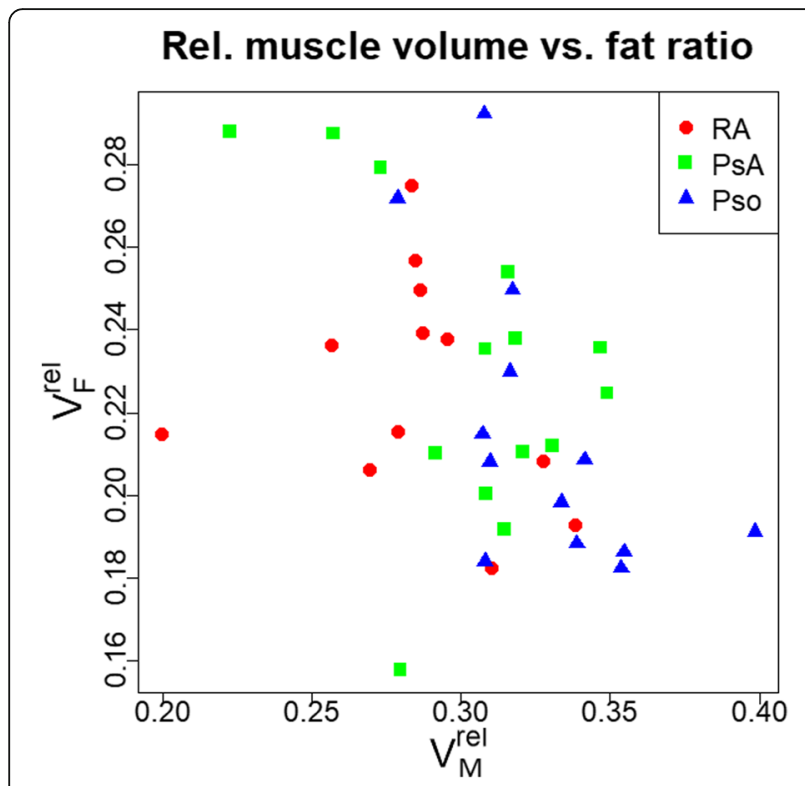

Fig. 8 Fat ratio plotted against relative muscle volume for all groups and both sex

\section{Conclusions}

These preliminary data showed that relative hand muscle volume decreased with age in male and female patients with Pso, PsA and RA patients. Independent of age, relative hand muscle volume was smaller in patients with RA compared to the patients with Pso and the difference was twice as large in males compared to females. Also in male but not in female RA patients relative hand muscle volume was smaller than in PsA patients. Preliminary results further indicate that the decrease in relative hand muscle volume is associated with an increase of relative fat content.

\section{Abbreviations}

BMI: Body mass index; CSA: Cross sectional area; fat: $_{\text {fixon fat image; }}$

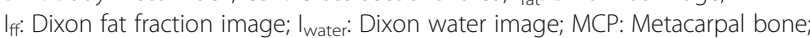
MRI: Magnetic resonance imaging; PsA: Psoriatic arthritis; Pso: Psoriasis; RA: Rheumatoid arthritis; RC: Rheumatoid cachexia; $\mathrm{S}_{\text {BMI: }}$ Subgroup of patients with available BMI data; $S_{\text {dixon }}$ : Subgroup of patients with available MR dixon images; TSE: Turbo spin echo; $V_{F}$ : Fat volume; $V_{F}^{\text {rel }}$ : Relative fat volume; $V_{H}$ : Hand volume; $V_{M}$ : Muscle volume; $V_{M}{ }^{\text {rel: }}$ : Relative muscle volume $\left(=V_{M} / V_{H}\right) ;$ VOI: Volume of interest

\section{Acknowledgements}

The present work was performed in partial fulfilment of the requirements for obtaining the PhD degree Dr. rer. Biol. hum. at the University of ErlangenNuremberg.

\section{Authors' contributions}

AF developed the software, analysed the datasets, performed the statistical analysis and created the original draft. CF, IdO, AK and DS collected data and reviewed and edited the original draft. AG implemented and tested the clinical hand MR Dixon protocol. TB, JR, MU and GS contributed to study design and patient investigations, provided data interpretation and reviewed and edited the original draft. GS furthermore contributed to the analysis and made major contribution in writing the manuscript. KE as senior author had designed the study, supervised the project and contributed to the manuscript. All authors read and approved the final manuscript.

\section{Funding}

This project has been funded by the German Federal Ministry of Education and Research, METARTHROS, BMBF 01EC1407A. The funding was used for software development and data analysis.

\section{Availability of data and materials}

The datasets generated and/or analysed during the current study are not publicly available since the consent does not include an agreement to put the patient images in public domain, but are available from the corresponding author on reasonable request.

\section{Ethics approval and consent to participate}

The patient consent form included an agreement to also use the data for research purposes (Ethics approval 52_14B of the Medical Faculty of FAU Erlangen-Nuremberg).

\section{Consent for publication}

Not applicable.

\section{Competing interests}

The authors declare that they have no competing interests.

\section{Author details}

${ }^{1}$ Institute of Medical Physics, University of Erlangen-Nuremberg, Henkestraße 91, 91052 Erlangen, Germany. ${ }^{2}$ FAU Erlangen-Nuremberg, Department of Internal Medicine 3, and Universitätsklinikum, Erlangen, Germany. ${ }^{3} \mathrm{FAU}$ Erlangen-Nuremberg, Radiological Institute, and Universitätsklinikum, Erlangen, Germany.

Received: 13 January 2019 Accepted: 9 March 2020

Published online: 02 April 2020

\section{References}

1. Challal S, Minichiello E, Boissier MC, Semerano L. Cachexia and adiposity in rheumatoid arthritis. Relevance for disease management and clinical outcomes. Joint Bone Spine. 2016;83(2):127-33.

2. Roubenoff $R$, Roubenoff RA, Ward LM, Holland SM, Hellmann DB. Rheumatoid cachexia: depletion of lean body mass in rheumatoid arthritis. Possible association with tumor necrosis factor. J Rheumatol. 1992;19(10): 1505-10.

3. Lemmey AB. Rheumatoid cachexia: the undiagnosed, untreated key to restoring physical function in rheumatoid arthritis patients? Rheumatology. 2016;55(7):1149-50.

4. Roubenoff R. Rheumatoid cachexia: a complication of rheumatoid arthritis moves into the 21st century. Arthritis Res Ther. 2009;11(2):108.

5. Masuko K. Rheumatoid cachexia revisited: a metabolic co-morbidity in rheumatoid arthritis. Front Nutr. 2014;1:20.

6. Summers GD, Deighton CM, Rennie MJ, Booth AH. Rheumatoid cachexia: a clinical perspective. Rheumatology. 2008;47(8):1124-31.

7. Marcus RL, Addison O, Kidde JP, Dibble LE, Lastayo PC. Skeletal muscle fat infiltration: impact of age, inactivity, and exercise. J Nutr Health Aging. 2010; 14(5):362-6.

8. Visser M, Kritchevsky SB, Goodpaster BH, Newman AB, Nevitt M, Stamm E, Harris TB. Leg muscle mass and composition in relation to lower extremity performance in men and women aged 70 to 79 : the health, aging and body composition study. J Am Geriatr Soc. 2002;50(5):897-904.

9. Frontera WR, Hughes VA, Fielding RA, Fiatarone MA, Evans WJ, Roubenoff R. Aging of skeletal muscle: a 12-yr longitudinal study. J Appl Physiol. 2000; 88(4):1321-6.

10. Arner $E$, Ryden $M$, Arner P. Tumor necrosis factor alpha and regulation of adipose tissue. N Engl J Med. 2010;362(12):1151-3.

11. Hotamisligil GS, Shargill NS, Spiegelman BM. Adipose expression of tumor necrosis factor-alpha: direct role in obesity-linked insulin resistance. Science. 1993;259(5091):87-91.

12. Veale D, Yanni G, Rogers S, Barnes L, Bresnihan B, Fitzgerald O. Reduced synovial membrane macrophage numbers, ELAM-1 expression, and lining layer hyperplasia in psoriatic arthritis as compared with rheumatoid arthritis. Arthritis Rheum. 1993;36(7):893-900.

13. Foell D, Kane D, Bresnihan B, Vogl T, Nacken W, Sorg C, Fitzgerald O, Roth J. Expression of the pro-inflammatory protein S100A12 (EN-RAGE) in rheumatoid and psoriatic arthritis. Rheumatology. 2003;42(11):1383-9. 
14. Naredo E, Moller I, de Miguel E, Batlle-Gualda E, Acebes C, Brito E, Mayordomo L, Moragues C, Uson J, de Agustin JJ et. al. High prevalence of ultrasonographic synovitis and enthesopathy in patients with psoriasis without psoriatic arthritis: a prospective case-control study. Rheumatology. 2011;50(10):1838-1848.

15. Faustini F, Simon D, Oliveira I, Kleyer A, Haschka J, Englbrecht M, Cavalcante AR, Kraus S, Tabosa TP, Figueiredo C et. al. Subclinical joint inflammation in patients with psoriasis without concomitant psoriatic arthritis: a crosssectional and longitudinal analysis. Ann Rheum Dis 2016;75(12):2068-2074.

16. Tustison NJ, Avants BB, Cook PA, Zheng Y, Egan A, Yushkevich PA, Gee JC. N4ITK: improved N3 bias correction. IEEE Trans Med Imaging. 2010;29(6): 1310-20.

17. Sled JG, Zijdenbos AP, Evans AC. A nonparametric method for automatic correction of intensity nonuniformity in MRI data. IEEE Trans Med Imaging. 1998;17(1):87-97.

18. Criminisi A, Shotton J, Konukoglu E. Decision forests: A unified framework for classification, regression, density estimation, manifold learning and semisupervised learning. Foundations and Trends in Computer Graphics and Vision. 2012;7(2-3):81-227.

19. Cuingnet R, Prevost R, Lesage D, Cohen LD, Mory B, Ardon R. Automatic detection and segmentation of kidneys in $3 D C T$ images using random forests. In: International Conference on Medical Image Computing and Computer-Assisted Intervention: 2012. Springer; 2012;66-74.

20. Ma J. Dixon techniques for water and fat imaging. J Magn Reson Imaging. 2008;28(3):543-58.

21. Glüer C-C, Blake G, Lu Y, Blunt B, Jergas M, Genant H. Accurate assessment of precision errors: how to measure the reproducibility of bone densitometry techniques. Osteoporos Int. 1995;5(4):262-70.

22. Gunther CM, Burger A, Rickert M, Crispin A, Schulz CU. Grip strength in healthy caucasian adults: reference values. J Hand Surg Am. 2008;33(4):558-65.

23. The R Project for Statistical Computing. https://www.r-project.org/.

24. Fischer MA, Pfirrmann CW, Espinosa N, Raptis DA, Buck FM. Dixon-based MRI for assessment of muscle-fat content in phantoms, healthy volunteers and patients with achillodynia: comparison to visual assessment of calf muscle quality. Eur Radiol. 2014;24(6):1366-75.

25. Crawford RJ, Filli L, Elliott JM, Nanz D, Fischer MA, Marcon M, Ulbrich EJ. Age- and level-dependence of fatty infiltration in lumbar paravertebral muscles of healthy volunteers. AJNR Am J Neuroradiol. 2016;37(4):742-8.

26. Marcon M, Berger N, Manoliu A, Fischer MA, Nanz D, Andreisek G, Ulbrich EJ. Normative values for volume and fat content of the hip abductor muscles and their dependence on side, age and gender in a healthy population. Skelet Radiol. 2016;45(4):465-74.

\section{Publisher's Note}

Springer Nature remains neutral with regard to jurisdictional claims in published maps and institutional affiliations.

Ready to submit your research? Choose BMC and benefit from:

- fast, convenient online submission

- thorough peer review by experienced researchers in your field

- rapid publication on acceptance

- support for research data, including large and complex data types

- gold Open Access which fosters wider collaboration and increased citations

- maximum visibility for your research: over $100 \mathrm{M}$ website views per year

At BMC, research is always in progress.

Learn more biomedcentral.com/submissions 